\title{
Constraints on Mechanisms for Longitudinal Spreading of Impulsive SEPs from Multispacecraft Observations of Scatter-free Events
}

\author{
M. E. Wiedenbeck ${ }^{* i} a$, C. M. S. Cohen $^{b}$, A. Klassen ${ }^{c}$, R. A. Leske ${ }^{b}$, P. A. Liewer ${ }^{a}$, \\ G. M. Mason ${ }^{d}$, and N. V. Nitta ${ }^{e}$
}

a Jet Propulsion Laboratory, California Institute of Technology

Pasadena, CA 91109 USA

${ }^{b}$ California Institute of Technology

Pasadena, CA 91125 USA

${ }^{c}$ Christian-Albrechts Universität zu Kiel

D-24118 Kiel, Germany

d Applied Physics Laboratory, Johns Hopkins University

Laurel, MD 20723 USA

${ }^{e}$ Lockheed-Martin Solar and Astrophysics Lab

Palo Alto, CA 94304 USA

E-mail: mark.e.wiedenbeck@jpl.nasa.gov

\begin{abstract}
Impulsive solar energetic particle (ISEP) events are understood to involve particle acceleration in relatively compact regions of the solar corona where reconnection causes the release of magnetic energy and produces both turbulence and larger scale motions that can interact with and accelerate charged particles. In many cases the longitudinal spread of ISEPs observed at $1 \mathrm{AU}$ is relatively narrow and possibly consistent with a point source of acceleration. However, several ISEP events observed with the two STEREO spacecraft and near-Earth instruments have had exceptionally wide longitudinal spreads, sometimes significantly greater than $90^{\circ}$. It has been suggested that this spreading could be caused by interplanetary scattering in conjunction with corotation of the interplanetary field. There exists a subset of ISEP events that are referred to as "scatter free" due to characteristics such as velocity dispersion, strong particle anisotropy, and/or flux dropouts observed at 1 AU. We report on scatter-free events observed by both of the STEREOs in 2014 when the spacecraft were separated by $38^{\circ}$. Producing such a large spread in the absence of significant interplanetary scattering requires a process other than cross-field diffusion for the longitudinal transport and suggests that the spreading could have its origin in the solar corona.
\end{abstract}

The 34th International Cosmic Ray Conference,

30 July- 6 August, 2015

The Hague, The Netherlands

\footnotetext{
*Speaker.

†The JPL author's copyright for this publication is held by the California Institute of Technology. Government Sponsorship acknowledged.
} 


\section{Introduction}

It is recognized that there are two classes of solar energetic particle (SEP) events, which are commonly called "gradual" and "impulsive". In a 1999 review [1], Reames documented the observed characteristics of the two classes and attributed them to two different acceleration mechanisms, shock acceleration for gradual events and flare-related acceleration for impulsive events. Over the past 15 years that classification has provided a framework for a large body of SEP research that further refined the distinctions between the two classes and, in some cases, identified ambiguities. Besides the compositional characteristics [2], including strong enhancements of ${ }^{3} \mathrm{He}, \mathrm{Fe} / \mathrm{O}$, and electrons, that are commonly used in identifying impulsive events, one characteristic that distinguished impulsive from gradual events is the range of heliographic longitudes over which they typically are observed. Although gradual events have wide distributions, single-spacecraft studies of impulsive events in which associations were identified between impulsive SEPs detected at $1 \mathrm{AU}$ and x-ray flares on the Sun showed that the distribution of longitudinal offsets between the flare location and the particle detection has a mean value consistent with the offset associated with the Parker spiral magnetic field and a standard deviation $\sigma \simeq 20^{\circ}$ [3]. This distribution was attributed to particle acceleration in a small spatial region on the Sun with particles propagating into the heliosphere within a relatively narrow magnetic flux tube connected to that region.

With the launch of the twin spacecraft of NASA's Solar Terrestrial Relations Observatory (STEREO) mission in 2006, observations of individual SEP events from multiple locations near 1 AU became possible. A number of studies using data from STEREO-A (Ahead), STEREO-B (Behind), and near-Earth spacecraft are now providing new observational constraints on our understanding of the longitudinal characteristics of both impulsive and gradual events.

The solar-minimum years 2007 through 2009 had very few SEP events and by the time activity began to increase in 2010, STEREO-A and -B were already widely separated. By November 2008 when an impulsive event was detected, the separation between each STEREO and Earth was $\sim 40^{\circ}$ (i.e., about $2 \sigma$ from the mean of the expected longitude distribution $[1,3]$ ). By a year later the separation had increased to $\sim 60^{\circ}(3 \sigma)$. The detection of the 3-4 November 2008 event [4] in ${ }^{3} \mathrm{He}$ at STEREO-B and ACE and in electrons at all three spacecraft was surprising. The event of 7 February 2010, in which both ${ }^{3} \mathrm{He}$ [5] and electrons [6] were observed at the three spacecraft when they were distributed over $136^{\circ}$ in heliographic longitude (the maximum extent over which an impulsive event has been detected to date) made it apparent that there are some events that could not be adequately accounted for in the conventional model of impulsive event acceleration and transport.

Although numerous impulsive events have been observed at only a single spacecraft during the STEREO era [7], the widely spread, multispacecraft events have garnered considerable interest because they are difficult to understand from a model in which the accelerated particles are injected at a point on the Sun and propagate into the heliosphere within a relatively narrow magnetic flux tube. Giacalone \& Jokipii [8] investigated the possibility that the spread of $\mathrm{MeV}$ ions in the 7 February 2010 event could be due to interplanetary diffusion and found that they could account for the time-intensity profiles at the three spacecraft using a combination of a parallel mean free path of several tenths of an AU and a ratio between perpendicular and parallel diffusion coefficients of a few percent. They also emphasized contribution to the longitudinal spreading caused by 
corotation of the magnetic field frozen into the radially expanding solar wind. Their analysis did not attempt to distinguish between diffusion caused by interplanetary scattering of the particles and diffusion caused by the random walk of magnetic field lines.

In addition to spreading during propagation through the interplanetary medium, it is also possible that significant longitudinal transport could occur before release from the solar corona. Potential field source surface (PFSS) maps, which model the magnetic field connecting the photosphere to a "source surface" (typically treated as a sphere of radius 2.5 solar radii) beyond which all field lines are open out into heliosphere, frequently indicate that field concentrations at an active region can expand by tens of degrees in the corona [9]. However a search of 14 years of PFSS maps [5] showed that they essentially never include fields that undergo sufficient spreading in the corona to account for the width of the 7 Feb 2010 event.

In this study we have searched for multispacecraft, impulsive SEP events that have other characteristics that can be used to rule out one or more of the possible mechanisms for longitudinal spreading. In Section 2 we discuss the indicators that can be use to establish that interplanetary scattering has not significantly affected an event. Section 3 discusses observations of the event of 2 May 2014 in which such characteristics were present. It is also argued that the random walk of magnetic field lines is not sufficient to produce the observed longitudinal spread. Thus we conclude that the spreading in this event must have occurred close to the Sun. Section 4 relates the 2 May 2014 event to three other events that occurred around the same time and shows that the event detections can be understood from the PFSS map and the evolving magnetic connections to $1 \mathrm{AU}$. Section 5 contains additional discussion of the results.

\section{Scatter-free Events}

Events in which longitudinal spreading is caused by scattering in the interplanetary medium should differ from those in which particles are transported along field lines and spread due to the longitudinal expansion of the field lines themselves, either because the particles were distributed in longitude before leaving the corona or because they are tied to field lines whose footpoints have undergone a random walk. When there is minimal scattering, the arrival times at $1 \mathrm{AU}$ of particles released simultaneously at the Sun exhibit velocity dispersion, with high-energy particles that have field-aligned velocities arriving first and particles with lower energies and/or larger pitch angles arriving later (see, however, [10]). The result is a characteristic "swoosh" pattern of onset times as a function of particle energy. Although many impulsive events have been observed to have velocity dispersion, these events are in the minority.

Another phenomenon indicative of minimal interplanetary scattering is the occurrence of flux "dropouts", which are sudden decreases in particle intensities during the course of an event. When a dropout occurs during the swoosh portion of an event, one sometimes observes a return to the original dispersion curve when the dropout ends. This provides compelling evidence that the dropout was caused by the spacecraft moving from a flux tube that contained particles from the SEP event to an adjacent flux tube that did not and then moving back into the populated flux tube. The existence of sharp particle intensity gradients between adjacent flux tubes indicates that there is minimal cross-field scattering in the event. Examples of velocity dispersion and of dropouts are shown by Mazur et al. [11]. 
Measurements of angular distributions of the particles in an impulsive SEP event can also provide information about scattering conditions. If the particles have undergone numerous scattering interactions between the Sun and $1 \mathrm{AU}$ their angular distribution will approach isotropic. On the other hand, if there is minimal scattering the particles should be beamed along the field and have a significant anisotropy.

Using these indicators we have searched for "scatter-free" impulsive SEP events that are detected in ${ }^{3} \mathrm{He}$ at two or more spacecraft.

\section{The Scatter-free Event of 2 May 2014}

The best example to date of a scatter-free, multispacecraft impulsive SEP event was detected on 2 May 2014 at the two STEREOs. We have presented preliminary reports [12, 13] of our observations of ${ }^{3} \mathrm{He}$ in this event using the STEREO/LET instruments. In addition, Klassen et al. [14] studied this event in near-relativistic electrons using the STEREO/SEPT instruments and found short durations ( $\leq 12 \mathrm{~min}$ ) and strong anisotropy at both spacecraft. This was the first electron "spike" event detected by both STEREOs.

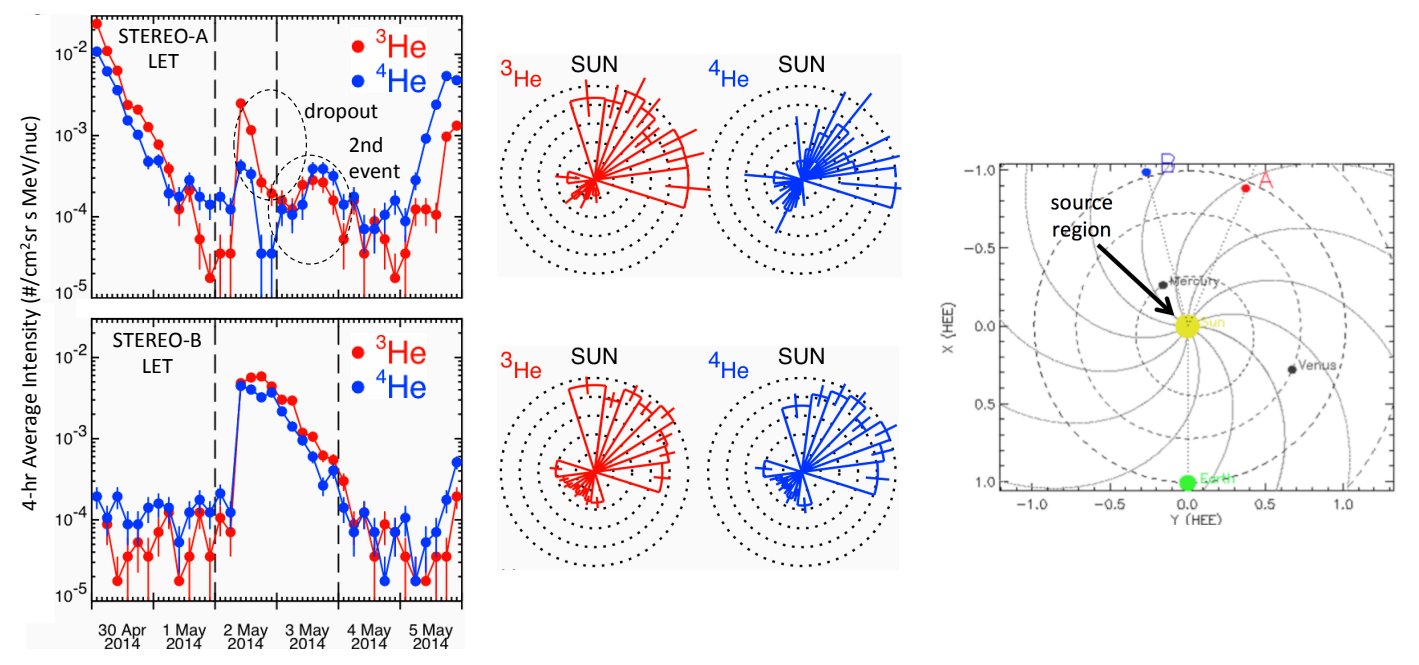

Figure 1: Left and center: Data from STEREO-A/LET (top) and STEREO-B/LET (bottom) for the 2 May 2014 event. The left-hand portion of the figure shows He isotope intensities as a function of time. The center portion shows angular distributions in the spacecraft frame, with the sunward direction at the top and the nominal sunward Parker spiral direction to the top-right. The angular-distribution data are integrated over the time interval bounded by vertical dashed lines in the intensity versus time plots. Right: Locations of the spacecraft and the active region on 2 May 2014.

The upper portion of Figure 1 shows 2.3-3.3 MeV/nuc He isotope data from STEREO-A/LET, with intensity versus time plots on the left and angular distributions on the right. The corresponding plots of STEREO-B/LET data are shown in the lower portion of the figure. At STEREO-B the intensity versus time curves have a sharp onset and an approximately exponential decay, as is often observed in SEP events. At STEREO-A, however, the event is cut off several hours after the initial rise. We interpret this as a flux dropout cause by STEREO-A leaving the flux tube along which 
the particles were propagating. The STEREO-A time profile is complicated by the presence of a second, weaker event on 3 May.

The angular distributions shown for the two spacecraft during the 2 May event include data from the time interval delimited by the dashed vertical lines on the intensity versus time plots. At both spacecraft the particle flows are away from the Sun with a strong front/back anisotropy.

Additional information can be obtained by looking at lower energy ion data available from the SEPT and SIT instruments. The left-hand panel of Figure 2 shows an energy spectrogram containing ion measurements from the SEPT instrument on STEREO-B. These ion data exhibit a clear swoosh pattern with a derived release time that is consistent with that obtained from the electron data [14]. The right-hand side of the figure shows spectrograms of ion data from the SIT instrument plotted as $1 / v$ versus time. In this representation a swoosh appears as a straight line. The plot from STEREO-B/SIT contains this pattern (indicated by the dashed red line that has been overplotted). This same line has been repeated on the STEREO-A/SIT plot, from which one can see that the ions were not observed at the Ahead spacecraft. By comparing with the STEREO-A/LET plot (Fig. 1) we conclude that the same dropout that caused the premature decrease of LET intensities was also responsible for the event not being observed in the lower-energy ions measured by SIT and by SEPT (not shown). The second event, seen by STEREO-A/LET on 3 May, is also evident as the start of another velocity dispersion curve at the far right of the plot for STEREO-A/SIT.
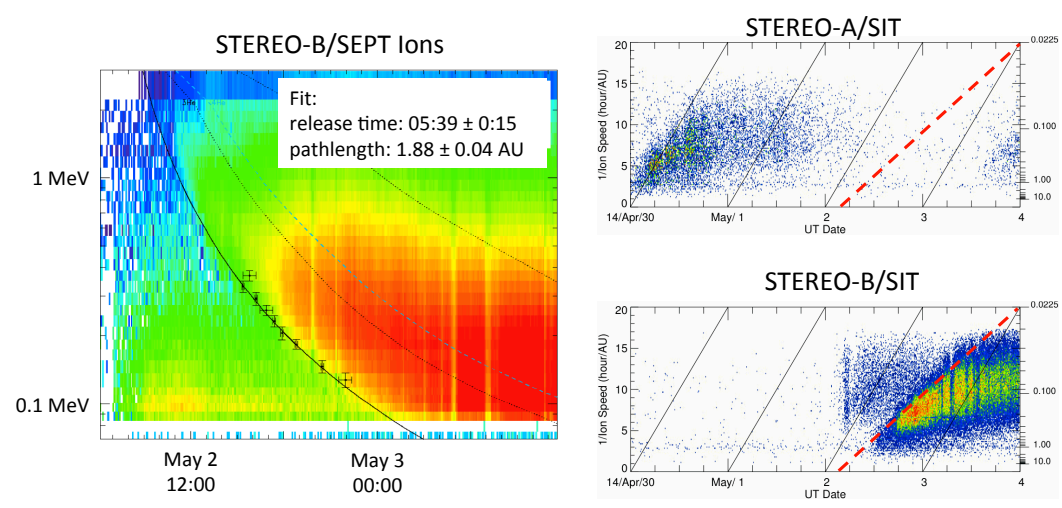

Figure 2: Ion data from SEPT (left) and SIT (right) show clear velocity dispersion. The right-hand vertical axis on the SIT plots indicates the energies (in MeV/nuc) corresponding to the inverse speeds shown on the left-hand axis. The dashed line in the upper right panel (copied from the lower right panel) shows where the event onset should have been observed if STEREO-A had not left the flux tube in which the particles were propagating around midday on 2 May.

\section{Other Events and the PFSS Map}

The impulsive event discussed above was the second in a series of four ${ }^{3} \mathrm{He}$-rich events detected by one or both of the STEREO/LET instruments during the period 29 April through 8 May 2014. The 29 May event was detected only at STEREO-A, the 2 May and 5 May events at both spacecraft, and the 8 May event only at STEREO-B. Figure 3 shows GONG potential field source surface (PFSS) maps superimposed on synoptic magnetograms for the four days. The PFSS maps 
show field lines that connect the ecliptic plane at the source surface to the photosphere. A star has been drawn on each of the maps at a point from which the plotted field lines expand to cover $\sim 95^{\circ}$ of heliographic longitude along the ecliptic plane. This is the approximate location at which STEREO/EUVI data indicate activity at the release time of the SEPs, as derived from electron and type III radio burst data in the first three events. The source location for the 8 May event is unclear because neither an electron event nor a type III burst was observed. We tentatively assume that the same region is responsible for this event. In this presentation, where the spacecraft locations and their footpoints at the Sun are nearly constant, the source region progresses from one panel of the plot to the next by about $40^{\circ}$ due to the rotation of the Sun. According to the PFSS map, the source region should have been connected to STEREO-A on 29 April but not to STEREO-B. On 2 May and 5 May it was connected to both spacecraft, and on 8 May it was only connected to STEREO-B. These connections would explain which spacecraft detected each event if our identification of the same active region as the source of all four events is correct. Furthermore, this region would not have been connected to either of the STEREO spacecraft before 29 April or after 8 May, consistent with the lack of additional ${ }^{3} \mathrm{He}$-rich event detections in this series.

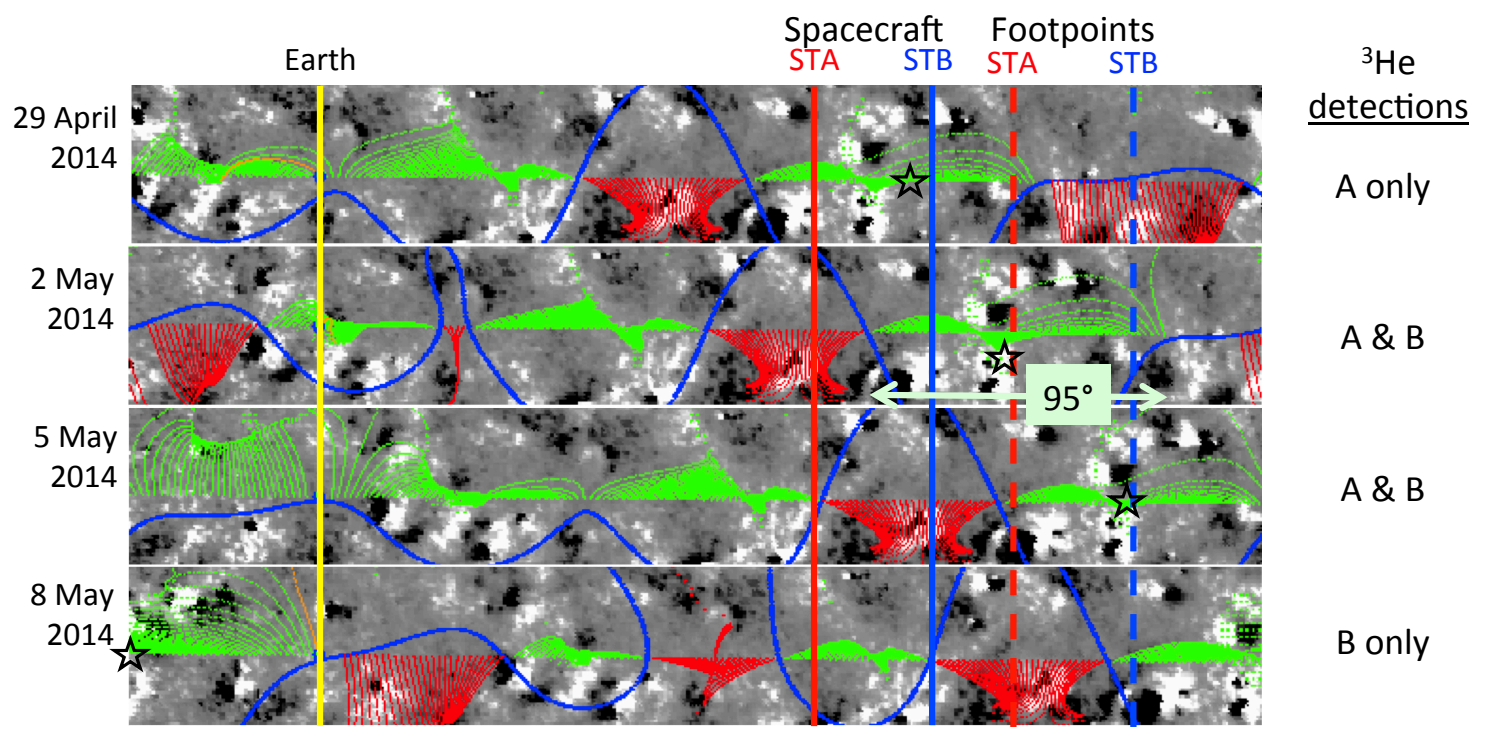

Figure 3: Potential field source surface maps overlaid on synoptic magnetograms for 4 days on which ${ }^{3} \mathrm{He}-$ rich SEP events were detected by one or both of the STEREO/LET instruments in late April to early May 2014. Solid vertical lines have been added to show the longitudinal locations of Earth (yellow), STEREO-A (red), and STEREO-B (blue). For the STEREOs dashed vertical lines are also shown indicating the nominal location of the magnetic footpoint at the Sun. Black stars indicate the location of the source region.

\section{Discussion}

The 2 May $2014{ }^{3} \mathrm{He}$-rich event was observed at both STEREO spacecraft in spite of the fact that they were separated by $38^{\circ}$ in heliographic longitude. The presence of velocity dispersion, a flux dropout at STEREO-A, and strong anisotropies all indicate that scattering by interplanetary turbulence cannot account for the longitudinal spread. Longitudinal transport without cross-field 
diffusion could occur either because the field lines disperse between the photosphere and the source surface, between the source surface and $1 \mathrm{AU}$, or both. Giacalone (private communication, 2015) has estimated that the random walk of field lines associated with footpoint diffusion driven by supergranular motions can account for at most $\sim 5^{\circ}$ degrees of spreading at $1 \mathrm{AU}$.

The PFSS model indicates that the source region for the 2 May event was connected to a wide range $\left(\sim 95^{\circ}\right)$ of heliographic longitudes along the ecliptic plane and that the footpoints of the magnetic field lines connecting to both STEREO-A and STEREO-B were within this region. STEREO/EUVI data indicate that the same active region was producing jets, which are commonly associated with impulsive SEP event acceleration, over an extended period in late April and early May, making this active region the likely source for the three additional ${ }^{3} \mathrm{He}$-rich events observed between 29 April and 8 May. The evolving connections to the two spacecraft as the Sun rotated can explain which spacecraft observed the different events. Thus we think it is likely that the longitudinal spreading during this time period was dominantly due to magnetic field line expansion between the photosphere and the source surface, with minimal additional longitudinal transport in the interplanetary medium.

The combination of a flaring active region connected to a wide range of heliographic longitudes by the field expanding in the corona and nearly scatter-free interplanetary transport are probably not a particularly common occurrence. Thus the interpretation of the longitudinal spread of the energetic particles observed in the 2 May 2014 event may not be generally applicable to other impulsive events. Indeed, the analysis of 14 years of daily PFSS maps [5] found that cases in which the field line spreading between the photosphere and the source surface could account for the largest longitudinal spreads observed in impulsive SEP events are exceedingly rare. Furthermore, the fact that 2 May 2014 was the first time that an electron spike event was observed at both STEREO spacecraft [14] shows that the conditions prevailing at the time were exceptional.

More commonly it may be the case that the wide longitudinal spreads observed in some impulsive events result from a combination of mechanisms including field-line spreading between photosphere and the source surface, random walk of field lines between the Sun and $1 \mathrm{AU}$, and cross-field scattering by interplanetary turbulence. It will be important to identify observational characteristics that can be used to determine the relative importance of these mechanisms. In addition to the features used to identify scatter-free events, we note that a strong falloff of event fluences with increasing distance from the well-connected field line has been reported in several ${ }^{3} \mathrm{He}$-rich events that have wide longitudinal spreads $[4,5]$. This probably reflects the diffusive transport across the field due to scattering on interplanetary turbulence. In contrast, in the 2 May 2014 event the peak intensities ${ }^{1}$ at both STEREOs were comparable in spite of the fact that STEREO-A was well connected to the source region and STEREO-B was separated by nearly $40^{\circ}$. We attribute this lack of a strong longitudinal dependence of the peak intensity to the dominance of field line spreading close to the Sun as the longitudinal transport mechanism in this event.

The NASA Solar Probe Plus and the ESA Solar Orbiter missions, which are now under development, will observe SEP events much closer to the Sun and provide additional constraints on particle transport in impulsive SEP events.

\footnotetext{
${ }^{1}$ For the 2 May 2014 event a more reliable comparison can be made between peak intensities than between fluences because of the dropout that occurred at STEREO-A shortly after the peak.
} 


\section{Acknowledgments}

We thank Joe Giacalone for his comments on field line random walk. This work was supported by NASA at JPL, Caltech, APL, and LMSAL. The STEREO/SEPT projects are supported under Grant 50 OC 1302 by the German Bundesministerium für Wirtschaft through the Deutsches Zentrum für Luft- und Raumfahrt (DLR).

\section{References}

[1] D. V. Reames, Particle Acceleration at the Sun and in the Heliosphere, Space Sci. Rev., 90, 413, 1999.

[2] G. M. Mason, ${ }^{3}$ He-Rich Solar Energetic Particle Events, Space Sci. Rev., 130, 231, 2007.

[3] D. V. Reames, Solar Energetic Particles: A Paradigm Shift, Rev. Geophys. Suppl., 33, 585, 1995.

[4] M. E. Wiedenbeck et al., Observations of a ${ }^{3}$ He-rich SEP Event over a Broad Range of Heliographic Longitudes: Results from STEREO and ACE, in proceedings of the Twelfth International Solar Wind Conference, AIP, CP1216, 621, 2010.

[5] M. E. Wiedenbeck et al., Observations of Solar Energetic Particles from ${ }^{3}$ He-rich Events over a Wide Range of Heliographic Longitude, ApJ, 762, 54, 2013.

[6] N. Dresing et al., Statistical Survey of Widely Spread Out Solar Electron Events Observed with STEREO and ACE with Special Attention to Anisotropies, A\&A, 567, A27, 2014.

[7] C. M. S. Cohen et al., Furthering Our Understanding of Wide Longitude ${ }^{3}$ He-rich SEP Events, Proc. 33rd Internat. Cosmic Ray Conf. (Rio de Janeiro), paper 802, 2013.

http: //www.cbpf.br/ icrc2013/proc_icrc2013.html

[8] J. Giacalone \& J. R. Jokipii, The Longitudinal Transport of Energetic Ions from Impulsive Solar Flares in Interplanetary Space, ApJL, 751, L33, 2012.

[9] P. C. Liewer, M. Neugebauer, \& T. Zurbuchen, Characteristics of Active-Region Sources of Solar Wind near Solar Maximum, Solar Phys., 223, 209, 2004.

[10] A. Sáiz et al., On the Estimation of Solar Energetic Particle Injection Timing from Onset Times near Earth, ApJ, 626, 1131, 2005.

[11] J. E. Mazur et al., Interplanetary Magnetic Field Line Mixing Deduced from Impulsive Solar Flare Particles, ApJL, 532, L79, 2000.

[12] M. E. Wiedenbeck et al., A Comparison of the Characteristics of ${ }^{3}$ He-Rich Solar Energetic Particle Events Observed at Single and at Multiple Spacecraft, AGU Fall Meeting, abstract SH21B-4116, 2014.

[13] M. E. Wiedenbeck et al., Scatter-free Impulsive SEP Events Observed at Multiple Spacecraft, First Joint AAS/AGU Triennial Earth-Sun Summit, abstract 401.03, 2015.

[14] A. Klassen et al., First Simultaneous Observations of a Near-relativistic Electron Spike Event by Both STEREO Spacecraft, A\&A, doi:10.1051/0004-6361/201525700, 2015. 